Voix et Images

voixetimages

\title{
Du bon usage des universités
}

\section{Robert Major}

Volume 21, numéro 2 (62), hiver 1996

Suzanne Jacob

URI : https://id.erudit.org/iderudit/201246ar

DOI : https://doi.org/10.7202/201246ar

Aller au sommaire du numéro

Éditeur(s)

Université du Québec à Montréal

ISSN

0318-9201 (imprimé)

1705-933X (numérique)

Découvrir la revue

Citer cet article

Major, R. (1996). Du bon usage des universités. Voix et Images, 21(2), 364-372.

https://doi.org/10.7202/201246ar d'utilisation que vous pouvez consulter en ligne.

https://apropos.erudit.org/fr/usagers/politique-dutilisation/ 
Essai

\section{Du bon usage des universités}

\section{Robert Major, Université d'Ottawa}

À quoi sert encore une université? On le savait, autrefois. L'Église québécoise le savait assez pour s'en méfier comme d'un lieu de libre pensée qu'il fallait chercher à contrôler. L'élite politique le savait tout autant, qui y envoyait ses rejetons pour y apprendre à penser et y acquérir ce vernis de culture sans lequel on ne saurait prétendre diriger le monde avec élégance ou une souveraine bonne conscience. Mais maintenant? 
Alors que les universités sont des usines à parchemins dont l'utilité est problématique tant ces diplômes sont dévalués par leur prolifération et les emplois devenus aléatoires? Au xIx ${ }^{\mathrm{e}}$ siècle, le cardinal Newman, écrivain et intellectuel influent, pouvait s'interroger longuement, dans son essai L'Idée d'une université, sur l'emplacement de ce lieu de haut savoir, analysant l'effet subtil de l'harmonie naturelle et d'un décor enchanteur. La fonction de l'université, comme lieu de pensée, de réflexion, d'échange, de rencontres, de fécondation réciproque, d'un Savoir à transmettre et à enrichir, allait de soi. Il pouvait donc s'attarder à des aspects plus ténus, tout comme Rabelais qui, quelques siècles plus tôt, avait conçu son abbaye de Thélème, site enchanteur favorisant le commerce des âmes bien nées.

Les choses, on le sait, ont bien changé, même s'il en est encore, paraitt-il, qui croient que les universités sont des tours d'ivoire, isolées du monde, et que les intellectuels qui les habitent ou arpentent leurs couloirs sont des êtres désincarnés, détachés du quotidien et quelque peu irresponsables. L'image est ancienne, et elle est coriace. Déjà Aristophane, dans Les Nuées, se faisait un malin plaisir de représenter Socrate, modèle, à ses yeux, des sophistes et précurseur de tous les professeurs, dans un "Pensoir", corbeille suspendue, flottant ainsi au-dessus des contingences et discourant à tout venant. Aristophane, doit-on le dire, était un traditionaliste qui déplorait l'individualisme effréné qui sévissait à son époque, et se faisait fort d'attaquer le rationalisme sceptique et opportuniste des sophistes dont l'enseignement bouleversait les valeurs et dévergondait la jeunesse.

Aujourd'hui aussi l'université est mise en cause et par tout un chacun, pas seulement les réactionnaires: les politiques qui voudraient qu'elle fasse ses frais, les néo-libéraux qui voudraient davantage l'arrimer au monde des affaires et à l'économie, les étudiants qui se disent déçus par la valeur d'échange de leurs diplômes, les professeurs qui s'interrogent sur les diverses fonctions dont ils se sentent investis. Il n'y a pas si longtemps, le livre d'Allan Bloom sur le sort fait à la culture générale dans les universités américaines avait fait ressortir avec éclat la démission de celles-ci face aux assauts du monde et leur ultime décomposition en de multiples discours hétéroclites ${ }^{1}$. Allan Bloom s'adressait à un vaste public. Or, voici qu'un livre récent s'adresse particulièrement aux professeurs et plus précisément aux spécialistes des sciences humaines.

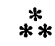

Le propos de Michel Freitag est donné dès le titre percutant de son recueil d'essais: Le Naufrage de l'université ${ }^{2}$. En effet, ce professeur de sociologie en a long à dire sur sa discipline et sur les "sciences humaines" 
en général. L'introduction du recueil ("La gestion technocratique du social") pose le diagnostic, fort sévère. L'époque actuelle ne nous demande pas "de prendre place dans un ordre pratique et symbolique déjà établi, d'être fidèles à des valeurs substantiellement définies, ni même de tendre vers la réalisation d'un idéal" (p. 9). On ne nous demande que de nous adapter au changement et de participer au mouvement. Mouvement vers quoi? Vers un futur toujours plus emballé, pauvre ersatz de l'Avenir qui, lui, était promesse.

Maintenant, la société ne se cache plus derrière un dieu, une idée, ou même une raison pour agir sur soi, et elle.a pour l'essentiel perdu la foi dans la capacité collective de faire l'avenir réflexivement. Elle se contente de chevaucher toutes les forces qu'elle a produites et libérées, toutes les puissances de mise en mouvement issues en son sein mais qui lui sont devenues étrangères, toutes les formes d'autorégulation nées au milieu des tourbillons du laisser-faire et par lesquelles elle a fini par se laisser dominer en même temps qu'elle renonçait à s'appréhender elle-même comme une totalité douée d'uné existence symbolique, d'une valeur normative, d'une capacité expressive: en un mot, d'une essence et d'un sens propres. (p. 13)

Cette situation inédite tient en bonne partie à la trahison des clercs, à la démission des clercs. En effet, dans la modernité, ce sont les sciences sociales qui ont remplacé la philosophie et les humanités pour assumer la fonction de réflexion de la société sur elle-même et de l'individu sur luimême ainsi que sur ses rapports avec autrui et le monde. Or, les sciences sociales ont abdiqué, ont trahi. Elles se contentent maintenant de "produire le futur de manière bureaucratique et technocratique, à travers la prise en charge et la gestion systématique, programmatique et prévisionnelle du social, sa mise sous contrôle" (p. 17). Elles n'étudient plus pour éclairer. Elles sont devenues inhumaines, asociales et ahistoriques, purement opérationnelles et techniciennes.

Michel Freitag propose donc une prise de conscience d'abord, une résistance ensuite, et une reconstruction au sein des universités, afin que les sciences sociales retrouvent leur fonction de savoir, s'écartent du pouvoir, cessent d'être simplement un savoir-faire au service d'une société organisationnelle dont les seuls objectifs sont la gestion et le contrôle. Les sciences sociales doivent redevenir des disciplines de réflexion et de formation. En somme, des "humanités".

Certes, Habermas a dit que la société opérationnelle, organisationnelle et décisionnelle était aussi un monde de la liberté quotidienne des personnes. Mais ce n'est qu'illusion, selon Michel Freitag. Cette liberté est insignifiante; c'est celle des molécules d'un gaz dont le mouvement est "libre", libre de s'accentuer sous la pression et l'échauffement, mais' qui ne va nulle part. On a défini la liberté comme absence de contraintes, et "chacun court après son sens propre et la réalisation de sa puissance propre comme après son ombre" (p. 21), car on a oublié que le sens est 
d'abord un "sens commun". La vie quotidienne a cessé de trouver place dans quelque chose qui puisse lui conférer un sens en la situant.

Un tel diagnostic social posé, il est entendu que la responsabilité des universités sera lourde et leur inculpation, menée sévèrement. Effectivement, dans l'essai éponyme du recueil, l'auteur se livre à une charge impitoyable contre l'orientation prise par les universités occidentales sous l'influence du modèle américain. Le seul objectif est une formation technicienne, pragmatique, axée sur l'emploi, orientée par les besoins de l'économie. Les fonctions traditionnelles de synthèse critique des connaissances et de libre recherche du Savoir ont été perdues. Certes, on brandit toujours les mêmes étendards: Savoir, Connaissance, Science, et recherche de la Vérité, mais ce sont des mots vides de sens. On ne veut connaître que les méthodes, les moyens, les techniques, et fournir le parchemin qui peut donner accès à l'emploi. Plus de synthèse, plus de compréhension critique, plus de véritable éducation, seulement des techniques.

Ce détournement se cristallise dans la fonction "recherche" des universités contemporaines, de plus en plus coupée de l'enseignement. On ne veut plus de maîtres, détenteurs d'un savoir, érudits dans leur domaine, capables de synthèse, doués pour la vulgarisation. On veut des professeurs-chercheurs (le professeur tout court n'étant qu'un amputé), dont le mandat sera la "formation des chercheurs", s'activant sur un savoir en miettes. "Tout le monde doit "chercher", non pas quelque chose, mais sur quelque chose." (p. 45) "La recherche n'est plus nulle part une recherche de savoir, de connaissance, de compréhension et de sagesse. Elle n'est plus associée à la patiente édification d'un idéal humain." (p. 52) "Pour résumer en quelques mots - qui sont excessifs bien sûr, mais comment répondre autrement à l'excès de la réalité qui nous étouffe? - , je dirai que dans l'immense majorité de toutes nos recherches, nous ne cherchons rien qui ait valeur de connaissance." (p. 53) La recherche a pris sens pour elle-même, car elle est comptabilisable, gérable et répond merveilleusement bien aux impératifs de la société actuelle. Mais elle s'est ainsi affranchie de toute finalité culturelle ou civilisationnelle. La recherche est à la fois la doctrine et la méthode, le moyen et la fin. Elle est science, parce qu'elle est méthodique.

Mais Michel Freitag pose brutalement la question: Aristote faisait-il de la recherche? Et Hume, Locke, Kant, Hegel, Nietzsche? "Mille informations ponctuelles ne valent pas une connaissance, mille décisions ne font pas un seul jugement synthétique, mille sondages ne donnent pas la prévisibilité d'une seule loi, mille probabilités ne confèrent pas la certitude d'un seul principe, mille descriptions ne font pas un unique concept. "(p. 57)

Un changement de cap radical s'impose donc, afin d'éviter le naufrage. L'université doit retrouver son sens premier et sa plus haute 
mission, se recentrer sur un "projet d'éducation supérieure d'orientation humaniste" (p. 59). Ainsi, la recherche universitaire doit viser le développement de "corps synthétiques de connaissances" (p. 64), être animée par "cet effort de synthèse compréhensive qui correspond à l'idée classique de la théorie" (p. 65). Quant aux recherches pragmatiques, utilitaires, visant des informations ponctuelles, elles doivent être laissées aux instances extra-universitaires. Dans la même logique, l'université doit engager des enseignants qui soient maîtres de leur discipline et capables de la transmettre; doit recréer des milieux vivants où la connaissance synthétique est valorisée; doit viser, non la formation de chercheurs sur un savoir fragmenté, mais une réflexion d'ordre philosophique et scientifique sur un savoir maitrisé.

On comprendra que de tels propos vaudront certainement à leur auteur l'accusation d'être un élitiste, un traditionaliste nostalgique, voire un réactionnaire impénitent. D'autant plus que les maîtres mots de ces essais sont "signification", "valeur", "sens", "idéal", enseignes qui s'opposent à "technocratique", "bureaucratique", "opérationnel ", "pragmatique", "organisationnel ", "systémique ", "autorégulation", "gestion", ces derniers termes étant quelquefois fusionnés, d'ailleurs, dans des raccourcis éloquents, comme celui qu'on trouve dans l'essai consacré à "La crise des sciences sociales": "mécanismes organisationnels et technobureaucratiques" (p. 74). Il s'agit moins d'une langue de bois que d'un procédé rhétorique d'inculpation.

D'ailleurs, il est sûr que cet auteur est en réaction à une situation qu'il juge alarmante, non seulement pour les disciplines universitaires, détournées de leur fin véritable, mais pour l'ensemble de la société, fortement menacée dans son existence même et dans sa survie par un développement emballé, échappant à tout contrôle normatif. Les sciences sociales, puisqu'elles ont usurpé une fonction réflexive sur la société en supplantant les humanités, doivent maintenant l'exercer, et pleinement. Elles doivent se livrer à une réflexion globale sur la société et son développement historique; retrouver une distance critique face aux instances qui cherchent à les cantonner dans des rôles de techniciennes au service d'un pouvoir diffus et englobant; réaffirmer la référence à des normes qui fondent ontologiquement la vie humaine et la condition sociale; lutter contre l'effritement et la dissolution de la société qu'on cherche à remplacer par un "social" détotalisé, dissolution que l'informatique et les médias accélèrent dramatiquement.

De tels propos confèrent au livre de Michel Freitag une acuité qui ne saurait nous laisser indifférents. On aurait tort, à mon avis, de n'y voir que simple vision crépusculaire, typique d'une angoisse fin de siècle (et de millénaire). Certes, comme littéraires, nous sommes partie prenante de sa réflexion: le rôle des universités nous concerne vivement, car nous en sommes; la spoliation des humanités par les sciences sociales, nous 
l'avons laissé faire, et l'avons même chaleureusement applaudie dans notre recherche effrénée de la scientificité; la "formation des chercheurs", nous l'avons faite nôtre, dans notre empressement à singer les "vraies" sciences; la recherche sur un savoir fragmenté et sans portée véritable, nous y sacrifions tous les jours dans notre poursuite d'une "excellence" mesurable. Mais il y a plus encore. Dans une société réduite au bavardage, à l'insignifiance, à la poursuite de petits bonheurs mesquins, le tout sous contrôle bureaucratique de plus en plus envahissant, une telle réflexion est devenue essentielle. Voici un livre de bonne foi qui n'attend que ses lecteurs, qu'on souhaite nombreux.

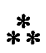

L'étude de Nicole Fortin, Une littérature inventée. Littérature québécoise et critique universitaire (1965-1975) ${ }^{3}$, prend un relief inattendu lorsqu'on la place en regard des propos de Michel Freitag. Il n'est plus question des sciences sociales, mais de ce qu'on appelait autrefois les "humanités", les études littéraires, c'est-à-dire les études qui - comme. chacun sait - ne sauraient prétendre au statut de science et qui, bien qu'elles soient, avec la philosophie, à l'origine même des universités, ne font pas le poids, actuellement, dans le cortège des disciplines sérieuses, celles dont une université se targue et qui font sa réputation auprès des bailleurs de fonds et des sondeurs.

Or, le phénomène qu'étudie Nicole Fortin illustre à merveille le propos de Michel Freitag: le rôle déterminant des universités dans l'éclosion de nouveaux discours et la participation empressée des professeurs dans la gestation de ce qui sera leur objet d'étude. La distance critique pourra bien repasser. Les jeunes critiques littéraires des années soixante avaient une mission et une passion : inventer la littérature québécoise.

Cette invention s'est effectuée dans de nombreux lieux, mais en particulier dans des revues universitaires pendant la décennie 1965-1975. Le point de départ de Nicole Fortin est donné dans la citation liminaire de François Ricard, selon lequel "sans la naissance d'une critique enfin compétente et tournée vers la vraie vie des œuvres, notre littérature n'aurait pu en si peu de temps franchir un si long pas" (p. 1). En effet, pendant ces années, il y avait, "à côté des romanciers, poètes, dramaturges, et de concert avec eux, la jeune génération des critiques et des professeurs, qui, dans leur domaine, déployèrent autant d'efforts et eurent autant d'enthousiasme que les artistes dans le leur, et contribuèrent donc aussi efficacement que ceux-ci, quoique de façon plus voilée, à l'extraordinaire fécondité de ces années" (p. 2).

La critique universitaire de ces années joue donc un rôle capital dans l'affirmation et dans la consolidation de la littérature québécoise. D'une 
part, elle accompagne les nouveaux textes qui prolifèrent et les impose à l'attention d'une nouvelle cohorte de lecteurs, instituant ces textes comme ceuvres et objets légitimes d'analyse; d'autre part, elle effectue des relectures de la littérature québécoise dans son ensemble, remet à jour un patrimoine, crée une tradition. Travail de redécouverte et de réappropriation tout aussi important que le premier. Sur un plan comme sur l'autre, au niveau de la contemporanéité comme de l'histoire, à l'échelle de la modernité comme à celle de la tradition, la critique fait connaître, diffuse, consacre.

Mais tout en contribuant à la fondation de la littérature, l'instance critique se fonde elle-même comme discours savant, occupant un lieu propre (les revues universitaires), et élaborant les règles discursives de son propre déploiement (scientificité, neutralité, rigueur) qui se veut en rupture avec la pratique antérieure, taxée de superficialité et d'émotivité facile, sinon de moralisme inepte. Les deux formations (formation du littéraire par la critique, formation de l'instance critique par son propre discours) se manifestent donc simultanément, en étroite interdépendance.

C'est d'ailleurs la deuxième formation qui intéresse particulièrement Nicole Fortin: l'analyse du discours, les modalités discursives, les théories argumentatives et axiologiques qui se déploient dans les trois revues savantes que sont Études françaises (1965- ), Voix et Images du pays (1967-1975) et Études littéraires (1968- ). Sans mettre en doute l'apport d'autres instances de légitimation et de consécration (journaux, revues culturelles et littéraires, etc.), elle s'en tient à ces lieux d'une pratique précise.

Mais l'analyse de ce lieu implique, du moins dans un premier temps, de négliger la spécificité de chacune des revues, comme d'ailleurs l'identité de chacun des intervenants, qui veulent être saisis dans une "sorte d'anonymat uniforme" (p. 25). Anonymat bien relatif, d'ailleurs, puisque les références de chacune des citations identifient bien chacun des intervenants. Ce qu'il faut comprendre par cette position, c'est que ce ne sont pas les propos d'un Gilles Marcotte, d'un Georges-André Vachon, d'un Jean Éthier-Blais ou d'un Robert Vigneault qui importent. C'est la stratégie discursive de, formalisation d'un savoir déployé par ces revues qui retient l'attention. L'analyse du champ discursif implique une saisie globale, pour mettre à jour les structures discursives et épistémiques communes. La spécificité de chacune des revues ne sera retrouvée que dans le dernier chapitre.

Auparavant, après une introduction et un premier chapitre qui examinent ces questions méthodologiques et interrogent le sens des termes employés (qu'est-ce que la littérature? la littêrarité? la québécité littéraire? la critique québécoise?), en puisant surtout, il me semble, à la sémiotique de Pierce, trois chapitres fort denses feront l'analyse du corpus retenu. 
Celui-ci est constitué des articles portant sur une problématique québécoise : 111 articles dans Études françaises, 112 dans Voix et Images du pays, 39 dans Études littéraires, vaste ensemble où il faut chercher les lieux communs ainsi que les jugements, idées et valeurs qui sont systématisés.

Le premier de ces chapitres examine le rapport à l'histoire, rapport ambigu ou problématique, car la critique littéraire, dans les années soixante, en raison de sa remise en question de l'histoire littéraire, tend à contester le rapport exclusif à la temporalité. Par ailleurs, la lecture de la littérature québécoise ne peut faire abstraction de la question de ses origines, de ses rapports avec la tradition, de ses diverses périodes et générations, du lien de causalité entre les textes, qui assure des axes de lisibilité ou de cohérence (évolution ou ruptures, par exemple). Le cas de Nelligan et celui de Saint-Denys Garneau sont particulièrement exemplaires de cette appartenance à des axes de lisibilité : à travers le temps, se construisent des lectures, et la critique universitaire des années soixante ne peut faire autrement qu'inscrire sa lecture dans un rapport de continuité ou de rupture avec celles qui l'ont précédée, comme elle ne peut négliger la question de l'appartenance de ces œuvres à un corpus d'attache.

Les chapitres suivants examinent, d'une part, la fondation du territoire, d'autre part, les fondements discursifs et cognitifs du discours critique. Le rapport à l'espace est celui du territoire référentiel (et sont alors convoqués les grands thèmes du pays et de l'exil qui ont marqué les textes et les lectures qui en ont été faites); mais il est aussi celui de l'espace discursif, la parole se déployant dans des marais de silence, la conscience s'éveillant dans un contexte d'aliénation. Par ailleurs, le dernier de ces trois chapitres analytiques portera sur le temps de la lecture, c'est-à-dire l'interdépendance féconde de la critique et de la littérature et leur création réciproque: le statut du texte, le statut du critique et de la critique.

Ces chapitres, comme le dernier où on rétablit la singularité de chacune des revues, témoignent d'un dépouillement rigoureux du corpus et d'une analyse tout à fait pertinente des articles. Certes, l'ensemble est quelque peu touffu et pas complètement dépourvu de redites; mais le volume nous présente un tableau très utile du discours littéraire de ces années charnières et même de fort profitables abrégés sur certaines problématiques (les interrogations sémantiques du premier chapitre, par exemple).

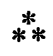

Peut-être pourrait-on rapprocher cette étude de Nicole Fortin de celle réalisée par son homonyme, universitaire à Laval également, et portant 
aussi sur des revues, plus nombreuses, toutefois, et réparties davantage dans le temps ${ }^{4}$. Dans les deux cas, les leitmotive de l'analyse sont la québécité et la modernité. Mais dans les deux cas, le lecteur (ce lecteur, du moins) ressent un certain malaise, qui ne tient pas à la qualité du dépouillement ni au sérieux du travail effectué, mais plutôt au caractère légèrement narcissique ou tautologique de l'entreprise. Des universitaires qui étudient le discours d'autres universitaires.

On sait que la littérature est de plus en plus faite par les universitaires (tant en ce qui concerne la création que les instances de production), qui changent ensuite de couvre-chef pour l'étudier, la leur et celle de leurs pairs. Et voici qu'on étudie le discours qu'ils tiennent sur celle-ci et qu'on leur confirme qu'ils sont co-inventeurs de cette littérature... Le tableau est de plus en plus chargé. À trop occuper le terrain, les universitaires ne sont-ils pas en train de tout étouffer? Ne resteront, bientôt, dans ce beau champ de plantes autofécondées, que beaucoup de tiges stériles, c'està-dire sans lecteurs autres que ces autres soi-mêmes. Michel Freitag a raison de souhaiter un peu de distance critique.

1. Allan Bloom, The Closing of the American Mind, publiê en français sous le titre: L'Âme désarmée. Essai sur le dêclin de la culture générale, Montréal, Guérin, 1987.

2. Michel Freitag, Le Naufrage de l'université. Et autres essais d'épistêmologie politique, Québec/Paris, Nuit blanche/La Découverte, coll. "Essais critiques", 1995, 301 p.

3. Nicole Fortin, Une littérature inventée. Littérature québécoise et critique universitaire (1965-1975), Sainte-Foy, Les Presses de l'Université Laval, coll, "Vie des lettres québécoises", 1994, $353 \mathrm{p}$.

4. Andrée Fortin, Passage de la moderntté. Les intellectuels québécois et leurs revues, Sainte-Foy, Les Presses de l'Université Laval, 1993, 406 p. Cette étude a été l'objet d'une brève analyse dans ma chronique de l'hiver 1994 (Voix et Images, $n^{\circ} 56$ ). 